\title{
Empirical Research Plan: Effects of Sketching on Program Comprehension
}

\author{
Sebastian Baltes ${ }^{1}$ and Stefan Wagner ${ }^{2(\bowtie)}$ \\ 1 University of Trier, Trier, Germany \\ research@sbaltes.com \\ ${ }^{2}$ University of Stuttgart, Stuttgart, Germany \\ stefan.wagner@informatik. uni-stuttgart.de \\ http://orcid.org/0000-0002-5256-8429
}

\begin{abstract}
Sketching is an important means of communication in software engineering practice. Yet, there is little research investigating the use of sketches. We want to contribute a better understanding of sketching, in particular its use during program comprehension. We propose a controlled experiment to investigate the effectiveness and efficiency of program comprehension with the support of sketches as well as what sketches are used in what way.
\end{abstract}

Keywords: Experiment $\cdot$ Sketching $\cdot$ Program comprehension

\section{Introduction}

Software is inherently abstract and has no natural representation except source code. Thus, especially for program comprehension, visualizations are important [1]. Sketches are an example for informal visualizations that are often created when understanding or explaining source code [2]. In the past, however, these informal artefacts did not get the amount of attention by the software engineering research community that their relevance in software development practice could imply. With our proposed study, we want to analyse if and how sketching improves program comprehension when explaining source code. Furthermore, we want to gain a better understanding of what sketches are used in what way to explain the source code. In the description of our experiment, we follow the guidelines of Jedlitschka, Ciolkowski and Pfahl [3].

\section{Related Work}

One of the main purposes of sketching in software development is communication $[2,4]$. To this end, developers often employ ad hoc notations that rarely adhere to standards like the Unified Modeling Language (UML) [2,5]. The ambiguity in sketches is a source of creativity [6] and they support problem solving and understanding [7]. In other areas like engineering, controlled experiments

(C) The Author(s) 2016

H. Sharp and T. Hall (Eds.): XP 2016, LNBIP 251, pp. 281-285, 2016.

DOI: $10.1007 / 978-3-319-33515-5 \_26$ 
have shown that the possibility to sketch has a positive effect on the quality of the solutions [8]. In our study, we want to analyse if sketches improve program comprehension in a setting where one developer explains a piece of source code to a colleague. To be able to compare the effect of sketching on program comprehension, we measure task correctness and response time [9].

\section{Experiment Planing}

The overall goal of our research is to better understand the use and usefulness of sketches in software engineering. In this experiment, we especially focus on sketching as a means of program comprehension in the communication between two developers. The goal of our experiment is:

Analyze sketching while explaining source code

for the purpose of evaluating its impact on program comprehension with respect to its effectiveness and efficiency

from the viewpoint of the developer

in the context of the conference XP 2016.

From this, we derive three research questions. The first two are more descriptive and exploratory to better understand which sketches developers use and how they use them while explaining source code to another developer. The third covers then the causal relationship of using sketches onto the effectiveness and efficiency of comprehending the source code.

RQ 1: Which sketches do developers use to explain code?

RQ 2: How do developers explain code with and without sketches?

RQ 3: How does the effectiveness and efficiency of the understanding of the code differ when it was explained with or without a sketch?

\subsection{Experimental Units and Materials}

The participants of the experiments will be pairs of developers. They will explain source code to each other. They have to be professional software developers.

We will use four different small open-source software systems in commonly known programming languages such as Java or C\#. As the developers do not know the source code beforehand but have to explain them, we limit the systems to $500 \mathrm{LOC}$ at most.

\subsection{Tasks}

The basic task for each pair of developers is to understand the source code of a small software system and then explain certain aspects to each other. The source code will be made available on an iPad. In case they should sketch, this will be done on paper. The aspects to explain will be low-level and code-centric. Afterwards, the developer the aspect was explained to, will answer questions evaluating how well they understood the explanations. 


\subsection{Hypotheses, Parameters and Variables}

The central independent variable of the experiment is the use of sketching. The dependent variables we are going to measure are the time needed until the explained aspect is understood and the correctness of the understanding. For the explorative part, we also document which types of sketches (e.g. different UML diagrams) they used and how they themselves judged the difference in explanations.

The two null hypotheses we are going to investigate are:

$\mathrm{H}_{01}$ : There is no difference in the effectiveness of comprehension with or without sketches.

$\mathrm{H}_{02}$ : There is no difference in the efficiency of comprehension with or without sketches.

Furthermore, we will document further context variables such as the experience of the developers with the programming languages and whether they have previously worked together.

\subsection{Experiment Design}

We will employ a blocked and balanced design. Hence, from each developer pair, the first developer will first read and explain a software system with sketching and then read another software system and explain it without sketching. The second developer will do the same but first without sketching and then with sketching.

We will openly invite the XP 2016 participants to join the experiment in pairs. Therefore, the sample is a convenience sample.

\subsection{Procedure}

We need a separate location for the experiment so that the participants can concentrate on understanding and explaining. We could hold it as one event during the conference or continuously over the whole conference depending on the fit to the conference schedule. We will put up lists in which the developers can volunteer to participate.

The first step when a pair starts the experiment is that they receive an iPad each with their two software systems to explain together with the question concerning the aspect they later have to explain to the other developer. Then (step 2) both get time to read the first system. In step 3, participant 1 explains the first system to participant 2 without a sketch. The time for this is measured on the iPad. Step 4 is a short questionnaire for participant 2 to check the correctness of their understanding. In step 5, participant 2 explains their software system aspect to participant 1 with the help of sketches on provided paper (including time measurement on the iPad). In step 6, participant 1 answers the short questionnaire concerning correctness.

Next, in step 7, both participants read the next question and source code. Then, the same procedure is repeated but participant 1 gets to use sketches while 
participant 2 does not. We will ask about the general experience and context factors in a final questionnaire.

\subsection{Analysis Procedure}

We will analyse the quantitative data to test the two hypotheses using an ANOVA analysis (RQ 3). Furthermore, we will qualitatively analyse the sketches and the answers to the open questions in the final questionnaire (RQ 1 and 2).

\section{Summary and Future Work}

In summary, we want to conduct a controlled experiment to better understand how developers use sketches in explaining source code as well as the effects on effectiveness and efficiency of the comprehension. The results of the experiment allow us to reduce the discrepancy between research concentrating on more formally defined modelling languages and the relevance of sketching in practice. Furthermore, we want to use the gained insights to work on a sketching language and tool support to aid practitioners in sketching in an efficient and effective way.

Open Access. This chapter is distributed under the terms of the Creative Commons Attribution-NonCommercial 4.0 International License (http://creativecommons.org/ licenses/by-nc/4.0/), which permits any noncommercial use, duplication, adaptation, distribution and reproduction in any medium or format, as long as you give appropriate credit to the original author(s) and the source, a link is provided to the Creative Commons license and any changes made are indicated.

The images or other third party material in this chapter are included in the work's Creative Commons license, unless indicated otherwise in the credit line; if such material is not included in the work's Creative Commons license and the respective action is not permitted by statutory regulation, users will need to obtain permission from the license holder to duplicate, adapt or reproduce the material.

\section{References}

1. Storey, M.D.: Theories, tools and research methods in program comprehension: past, present and future. Softw. Qual. J. 14(3), 187-208 (2006)

2. Baltes, S., Diehl, S.: Sketches and diagrams in practice. In: Proceedings of the International Symposium on Foundations of Software Engineering (FSE 2014), pp. 530-541 (2014)

3. Jedlitschka, A., Pfahl, D.: Reporting guidelines for controlled experiments in software engineering. In: International Symposium on Empirical Software Engineering (2005)

4. Cherubini, M., Venolia, G., DeLine, R., Ko, A.J.: Let's go to the whiteboard: how and why software developers use drawings. In: Proceedings of the SIGCHI Conference on Human Factors in Computing Systems (CHI 2007), pp. 557-566 (2007)

5. Petre, M.: UML in practice. In: Proceedings of the International Conference on Software Engineering (ICSE 2013), pp. 722-731 (2013) 
6. Goldschmidt, G.: The backtalk of self-generated sketches. Des. Issues 19(1), 72-88 (2003)

7. Suwa, M., Tversky, B.: External representations contribute to the dynamic construction of ideas. In: Hegarty, M., Meyer, B., Narayanan, N.H. (eds.) Diagrams 2002. LNCS (LNAI), vol. 2317, pp. 341-343. Springer, Heidelberg (2002)

8. Schütze, M., Sachse, P., Römer, A.: Support value of sketching in the design process. Res. Eng. Design 2(14), 89-97 (2003)

9. Dunsmore, A., Roper, M.: A comparative evaluation of program comprehension measures. Technical report EFoCS 35-2000, Department of Computer Science, University of Strathclyde (2000) 